The Seventh Asian Congress of Fluid Mechanics

Dec 8 - 12, 1997, Chennai (Madras)

\title{
NEW EVOLUTION EQUATIONS FOR TURBULENT BOUNDARY LAYERS IN ARBITRARY PRESSURE GRADIENTS
}

\author{
A.E. Perry 1. Marusic M.B. Jones \\ Department of Mechanical and Manufacturing Engineering, University of Melbourne, Parkville \\ VIC 3052, Australia.
}

\begin{abstract}
A new approach at looking at the classic closure problem for turbulent boundary layers is presented. This involves using the well known mean-flow scaling laws such as Prandtl's law of the wall and Coles' law of the wake together with the mean momentum integral and differential equations. The important parameters governing the flow in the general non-equilibrium case are identified and are used to formulate the closure hypothesis. Once the mean flow field has been determined, relevant turbulence quantities can be computed using a coherent structure eddy model based on the attached eddy hypothesis.
\end{abstract}

\section{INTRODUCTION}

In earlier initial work, Perry, Marusic \& Li (1994) derived a closure scheme for calculating the streamwise evolution of a turbulent boundary layer developing in a pressure gradient. The cases considered were not completely arbitrary and were confined to flows which could be considered to be in either approximate equilibrium or quasi-equilibrium. In approximate equilibrium flows, the Coles wake factor $\Pi$ is assumed to be constant and the shear stress profiles are characterised only by $\Pi$ (approximately) and in quasi-equilibrium flows, $\Pi$ is allowed to vary but this variation is sufficiently slow so as to keep the parameter $\zeta=S \delta_{c} d \Pi / d x$ small. Here $\delta_{c}$ is the boundary layer thickness and $S=U_{1} / U_{\tau}$ is a skin friction parameter where $U_{\tau}$ is the local friction velocity and $U_{1}$ is the local freestream velocity. This scheme was based in part on the law of the wall, law of the wake and the mean momentum differential and integral equations together with continuity. From the laws of the wall and wake and the momentum equation, the shear stress is found to be given by

$$
\frac{\tau}{\tau_{0}}=f_{1}[\eta, \Pi, S]+g_{1}[\eta, \Pi, S] \zeta+g_{2}[\eta, \Pi, S] \beta
$$

Here $\tau$ is total shear stress, $\tau_{0}$ is the wall shear stress, $\eta=z / \delta_{c}, z$ is the wall normal distance, $\beta$ is the Clauser pressure gradient parameter and $f_{1}, g_{1}$ and $g_{2}$ are known universal analytical functions. 
In the restricted flows considered by Perry et al. (1994), the parameter $\zeta$ is more or less neglected. Thus the problem reduced to considering the relation

$$
C[\Pi, \beta, S]=0
$$

where for a given $\Pi$ it is assumed that the defect distribution is fixed and the shear stress distribution is fixed. Hence from data, if we know $\beta$ at a given $S$ for a fixed $\Pi$ (ie. for one experimental data point), then for this fixed $\Pi$ we can find $\beta$ versus $S$ for all $S$ using equation (1) to ensure that $\tau / \tau_{0}$ profiles are matched for all S. Now, it is found that for $S$ sufficiently large, $\beta=\beta_{\mathrm{a}}$ (the asymptotic value of $\beta$ ) and $C$ is no longer a function of $S$. If this procedure is repeated for different values of $\Pi$, a one-to-one relationship between $\beta_{a}$ and $\Pi$ can be found which is based on experiment. This formulation is consistent with the usual universal relation for eddy viscosity $\varepsilon$, ie. $\varepsilon /\left(\delta_{c} U_{\imath}\right)=\phi[\eta, \Pi]$. Unfortunately, such formulations are known to break down severely in non-equilibrium flows such as that considered by Marusic \& Perry (1995).

\section{NEW FORMULATION}

In order to account for non-equilibrium flows, the parameter $\zeta$ must be added to our functional space and thus $C$ in (2) needs to be replaced by,

$$
F[\Pi, \beta, S, \zeta]=0
$$

Hence in order to describe the state of the layer, we require three of the four variables in the above expression. For quasi-equilibrium flows Perry et al. (1994) relied on a one-parameter family to describe the shear stress, ie. $\tau / \tau_{0}=f[\eta, \Pi]$ where $f$ is assumed to be universal. A two parameter family of shear stress profiles would be closer to the truth and when used in conjunction with (1), information can be obtained regarding (3) as follows. Consider the $S$ $\beta$ plane at a fixed $\Pi$. If such a plane contains an experimental data point, then $S, \Pi, \beta$ and $\zeta$ are known for that data point and so also is $\tau / \tau_{0}$ versus $\eta$ from (1). Trace out a curve for increasing $S$ of fixed shear stress profile shape on the $S-\beta$ plane by optimising $\zeta$ and $\beta$ for best least squares error fit to the $\tau / \tau_{0}$ versus $\eta$ profile. This can be done with very small error and is an improvement over the Perry et al. (1994) profile matching technique. Hence we can-compute $\zeta$ at various $S-\beta$ points for this fixed $\Pi$ along a curve of fixed shear stress profile shape. By taking $S \rightarrow \infty$ we obtain asymptotic values $\zeta_{\mathrm{a}}$ and $\beta_{\mathrm{a}}$. (Going to $S=\infty$ is simply a convenient curve-fitting procedure and could never be approached experimentally). If this process is repeated often enough for different $\Pi$ 's then we obtain a $\Pi-\beta_{\mathrm{a}}$ diagram with distributions of extrapolated data points corresponding to different values of $\zeta_{\mathrm{a}}$. By twodimensional curve-fitting we obtain an empirical relation of 


$$
\psi\left[\Pi, \beta_{\mathrm{a}}, \zeta_{\mathrm{a}}\right]=0
$$

By shear stress profile matching as indicated above, we can then map out isosurfaces of $\zeta$ in $\Pi-\beta-S$ space.

The two parameter family of shear stress profiles can be expressed as $\left.\tau / \tau_{0}=f \llbracket \eta, \Pi, \beta_{\mathbf{a}}\right]$ and with the aid of (3) formulated as above it turns out that the evolution equations can be reduced to two coupled ODEs in terms of $S$ and $\Pi$, thus

$$
\begin{aligned}
& \frac{d S}{d R_{x}}=\chi \phi_{1}\left[S, \Pi, \frac{1}{\chi^{2}} \frac{d \chi}{d R_{x}}\right] \\
& \frac{d \Pi}{d R_{x}}=\chi \phi_{2}\left[S, \Pi, \frac{1}{\chi^{2}} \frac{d \chi}{d R_{x}}\right]
\end{aligned}
$$

Here $\phi_{1}$ and $\phi_{2}$ are universal, $R_{x}=x U_{0} / v$ the streamwise Reynolds number, $U_{0}$ is the freestream velocity $U_{1}$ at an arbitrarily chosen "initial condition" point $x=0$ and $\chi=U_{1} / U_{0}$ and is expressed as a function of $R_{\mathbf{x}}$. For source and sink flows of strength $\mathbf{Q}$, it turns out that equations (5) and (6) become autonomous and solution trajectories can be displayed on the $S-\Pi$ phase plane. The source and sink flows are characterised by an acceleration parameter $K=\left(d \chi / d R_{x}\right) / \chi^{2}=v /\left(L U_{0}\right)=2 \pi v / Q$, see Fig. $1(a)$. Figures $1(b)$ and (c) shows some trial solution trajectories for a sink flow ( $\mathrm{K}$ positive) and for a zero pressure gradient layer $\mathrm{K}=0$ respectively. The quasi-equilibrium solution trajectories of Perry, Marusic \& $\mathrm{Li}$ (1994) are also shown.

Relationship (4) has been obtained experimentally and relies on very sparse data and this is likely to be so for some time. We are presently engaged in gathering a complete set of data for sink flow turbulent boundary layers but many more experiments are required in developing flows ( $\mathrm{d} \Pi / \mathrm{dx}$ positive) and relaxing flows ( $\mathrm{d} \Pi / \mathrm{dx}$ negative). Hence, so far, the formulation for (4) is very tentative. We hope to be guided further in formulating (4) with the aid of the attached eddy hypothesis as recently formulated by Perry \& Marusic (1995), who have successfully incorporated the concept of a wall structure and a wake structure. The approach is philosophically consistent with the integral method used here as it deals with the boundary layer as an "integrated whole" with the transport properties at one point being related to motions in regions remote from that point of interest. Once the mean flow field has been computed, the attached eddy model of Perry \& Marusic (1995) serves as a means of computing the broadband turbulent intensities, spectra and other relevant turbulence quantities.

Acknowledgments

The authors wish to thank the Australian Research Council for the financial support of this project. 


\section{References}

[1] Perry, A.E., Marusic, I. \& Li, J.D. Physics of Fluids. 6(2), 1024-1035, (1994).

[2] Marusic, I. \& Perry, A.E. J. Fluid Mech. 298, 389-407, (1995).

[3] Perry, A.E. \& Marusic, I. J. Fluid Mech. 298, 361-388, (1995)

(a)

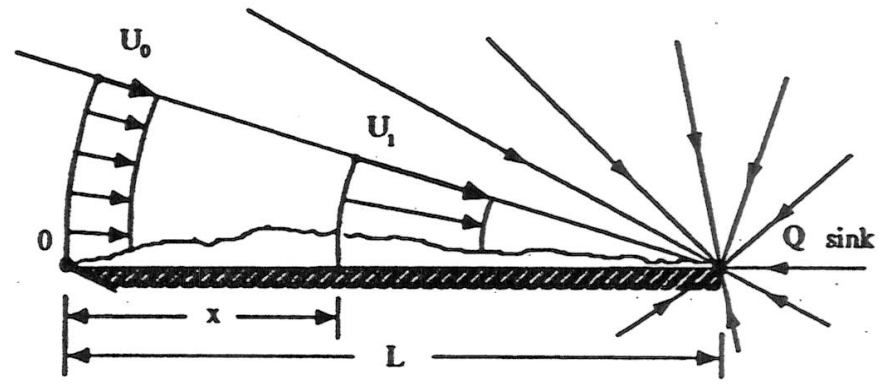

(b)

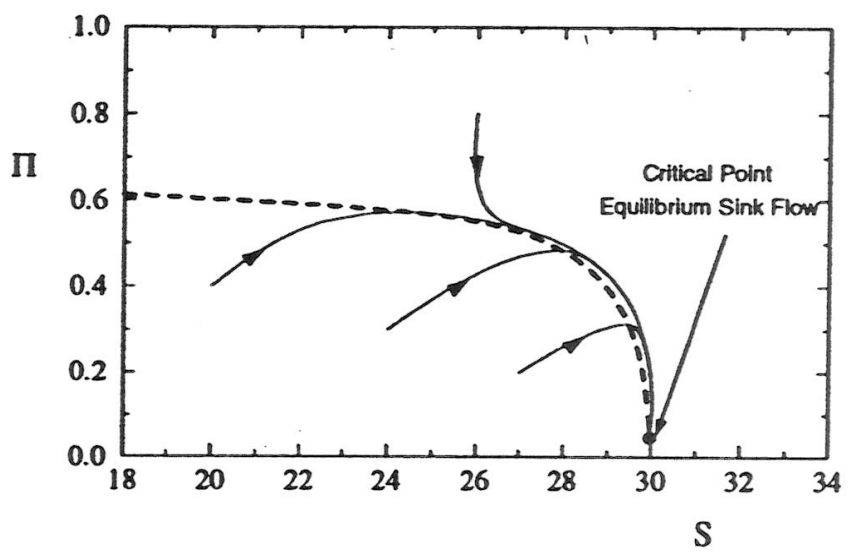

(c)

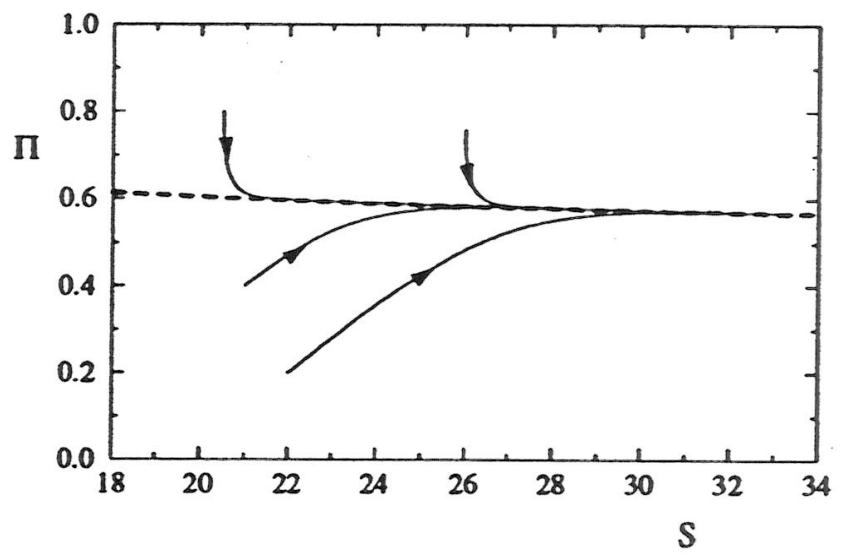

Fig. 1 Phase plane calculations. (a) Schematic of sink flow $\left(K=v /\left(L U_{0}\right)\right)$ with definitions, (b) $\mathrm{K}=10^{-8}$ sink flow, (c) $\mathrm{K}=0$, zero pressure gradient flow solution trajectories for different initial $\Pi$ and $S$ values. Broken lines are the quasi-equilibrium solutions where one is not free to choose arbitrary initial values of $\Pi$ and $S$ independently. 


\section{University Library}

\section{- M I N E R VA A gateway to Melbourne's research publications}

Minerva Access is the Institutional Repository of The University of Melbourne

Author/s:

Perry, A. E.;Marusic, I.;Jones, M. B.

Title:

New evolution equations for turbulent boundary layers in arbitrary pressure gradients

Date:

1997

Citation:

Perry, A. E. and Marusic, I. and Jones, M. B. (1997) New evolution equations for turbulent boundary layers in arbitrary pressure gradients, in Proceedings, The seventh Asian congress of fluid mechanics, Chennai (Madras) India.

Publication Status:

Published

Persistent Link:

http://hdl.handle.net/11343/34682 